\title{
Utilization of Antenatal care among pregnant women of Urban Slums of Dhaka City, Bangladesh
}

\author{
Russell Kabir ${ }^{1}$, Hafiz T.A.Khan ${ }^{2}$ \\ ${ }^{I}$ (Department of Economics and International Development/Middlesex University, UK) \\ ${ }^{2}$ (Department of Economics and International Development/ Middlesex University, UK)
}

\begin{abstract}
Maternal health services have a potentially critical role in the improvement of reproductive health. The use of health services is related to the availability, quality and cost of the services, as well as to social structure, health beliefs and the personal characteristics of the users. Antenatal care (ANC) is an important determinant of high maternal mortality rate and one of the basic components of maternal care on which the life of mothers and babies depend. It is a key strategy for reducing maternal mortality, but millions of women in developing countries do not receive it. Extracting 3549 women from the 2006-2007 Urban Health Survey, this study assessed the utilization of antenatal care and its related factor in urban slums for pregnant women. A Cross-sectional survey was carried out large, medium and small urban squatter settlements of Dhaka City. Two hundred married women in the age range 15-49 years were interviewed. Socio-demographic characteristics of women who received and who did not receive antenatal care in their previous pregnancy were assessed by frequency distribution and bivariate analysis. Antenatal care used in any of the previous pregnancy among women under study was $861(64.3 \%)$. Education and ANC is significantly related suggesting that the higher the level of education the higher is the likelihood of receiving ANC during pregnancy because educated women are aware about the importance of ANC during pregnancy. There is a strong association between the income of the respondents and ANC received by the respondents. This study indicates that overall knowledge about antenatal care was found to be better among women who had utilized antenatal care as compared to women who did not receive antenatal care.. There is also a need to evaluate the services provided by government health facilities and to find out why women are not utilizing the government health services though these services are available at subsidized rate.
\end{abstract}

Keywords - Antenatal Care, Mortality, Maternal health, Urban, Slum

\section{INTRODUCTION}

Millions of women in developing countries experience life threatening and other serious health problems related to pregnancy or childbirth. Complications of pregnancy and childbirth cause more deaths and disability than any other reproductive health problems [1]. This situation is worse in developing countries like Bangladesh due to inadequate access to modern health services and poor utilization. Despite the government serious commitment to deliver health facilities to the doorsteps of urban people through innovative approaches, such as Essential Service Package (ESP), the utilization of health service is still far below any acceptable standard [2]. Over the past decades, impressive studies have been made in reducing levels of infant and childhood mortality and increasing levels of contraceptive use in developing countries. In contrast, progress in reducing levels of maternal mortality and in making pregnancy and child bearing safer for women, despite being a central element of the millennium development goals, has been much slower [3]. Although exact figures remain elusive an estimated 525,000 women, almost all from developing countries, continue die each year from maternal causes [4].

In Bangladesh, antenatal care (ANC) usually refers to pregnancy related care provided by a health provider either in a medical facility or at home [5]. The necessities of ANC services by explaining the numerous advantages of them which may include monitoring health of the mother and baby during pregnancy, anticipating difficulties at pregnancy and labor with early treatment to reduce the risks for mother and child, facilitating the better use of emergency obstetric care services, disseminating health education and information, and so on [6]. These facilities imply that antenatal visits may confer benefits to both mother and baby, not only because women with serious conditions such as hypertensive diseases in pregnancy can be diagnosed and kept under observation, but also because a dialogue can be initiated which may facilitate the use of skilled care when the need arises [7].

Pregnancy and maternal health issue are culturally sensitive in Bangladesh. Like most of maternal health issues, proper care during pregnancy and childbirth is important to the health of both of the mother and child. Antenatal care is recognized as a major component of comprehensive maternal health care. It is the care that pregnant women should have during her pregnancy for protection of her and safe delivery. ANC is reachable through health service interventions. Antenatal care is essential for detection and treatment of 
problems during pregnancy and can improve the timely and appropriate use of delivery care services. A pregnant women needs to visit health facilities/providers at certain intervals for antenatal care check up. Evidence suggests that adequate antenatal care use has association with improved pregnancy outcome [8]. Due to rapid urbanization and industrialization so many women are coming from rural to urban areas for better opportunity and start living in slums and low economic conditions. The prevalence of reproductive health problems among women of reproductive ages in urban slums of Dhaka city is very high. Because of the poverty, low level of education and lack of accessibility health service utilization is very low. Women, especially poor women are often trapped in a cycle of ill health exacerbated by child bearing and hard physical labor. Many women in urban areas receive little nutrition during pregnancy. Timely antenatal check up has an impact in the reduction of maternal morbidity and maternal deaths. In the absence of not having proper antenatal check up may cause problem in pregnancy and various complications. To achieve MDG-5, reduction of maternal death will be an important strategy for Bangladesh. The present study would help in understanding the antenatal care utilization of urban slum poor women of Dhaka and would also identify the important factors related with antenatal care utilization.

\section{METHODOLOGY}

The study design was retrospective cross-sectional design. The study populations were pregnant women of urban slums of Dhaka city. Data of this study has been collected from Bangladesh Urban Health Survey (UHS) 2006. The survey was designed to obtain a broad health profile of the urban population in Bangladesh. This particular study only focused on the urban slum population of Dhaka city. The Urban Health Survey Interviewed ever married women from slums of Dhaka City. There were 3549 ever-married women of reproductive ages. Among them 1280 women had last one birth in the last five years. In the 2006 Urban Health Survey there were a total of eight domains. From each domain, 64 sample clusters (called primary sampling units: PSUs) were selected by probability proportional to size (PPS). Then about 25 households per cluster were selected by systematic random technique. In-depth interview through individual questionnaire for women were used for collection of information. All interview questionnaires were checked for their internal consistency to exclude missing or inconsistent data. Data entered in to the data file using statistical software called SPSS (Statistical Package for Social Science). Data checked, cleaned and edited properly before analysis. Data was analyzed in the SPSS and frequency distribution, Chi-Square and appropriate statistical test done for important variables. Each of the respondents were informed about the study and requested to provide verbal consent before starting the interview. To identify the factors that can influence the health seeking behavior of urban poor pregnant women related to ANC services, sexually transmitted diseases and morbidity of the children. It is expected that the findings would help policy makers, population scientists and planners to the understanding of health care utilization of urban slum poor women and can consider strategies for improving the health care utilization of the poor urban slum women. In retrospective study, it is difficult to get real picture of pregnant women and the associated factors because all related information were not available in the data set. Detailed information on maternal healthcare was available for the last births that have taken place during the last five years.

\section{RESULTS AND FINDINGS}

3.1 Socio-economic and demographic characteristics:

According to the sample characteristic, the mean age of the respondents was 30 years and about $16 \%$ women's age was below 20, $25 \%$ women's age was between 20-24 suggesting about $40 \%$ respondents were below 25 years. According to the educational standard 52.5\% respondents have ever attended school and rest $47.5 \%$ had never been to a school. Among those who attended school $54 \%$ had primary education followed by secondary and SSC passed 40.3\%. Only 4.9\% passed higher secondary and above shown in Figure 1.

Figure 1: level of education

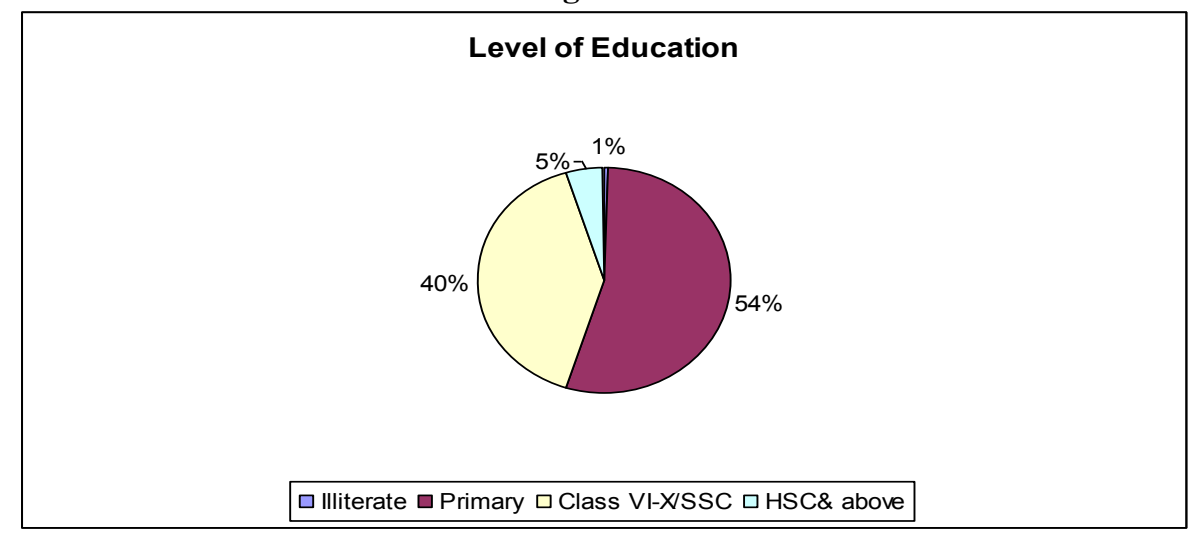


The median incomes of the respondents were Taka 1644 and Taka 1400 respectively. More than one third of the respondents (35.5\%) reported their family income was less than 5000 Taka and $43.5 \%$ of the respondents mentioned that family income was between 5000-10000. Only one in five respondents $(20.9 \%)$ reported that their family more than or equal to 10000 Taka.

The study results also shows that access to basic needs of the slum dwellers. Information on access to electricity, source of drinking water and access to toilet facility was also collected in the survey. As evident from the table, $95.4 \%$ of the households had access to electricity. Piped water (outside) is the predominant source of drinking water, which is $40.4 \%$. Only $12.2 \%$ household use tube well (inside) water and $33.5 \%$ household use piped water (inside). Only 15\% slum households had sanitary toilets (septic tank or modern toilet facility) at the time of survey; $40.7 \%$ households used open toilet indicating health hazards of the slum dwellers.

The respondents who were pregnant were asked whether they received ANC during the last pregnancy and the information gathered in this respect is shown in Figure 2 among the respondents $64.3 \%$ women received ANC during last pregnancy.

Figure 2: ANC Received in Last Pregnancy

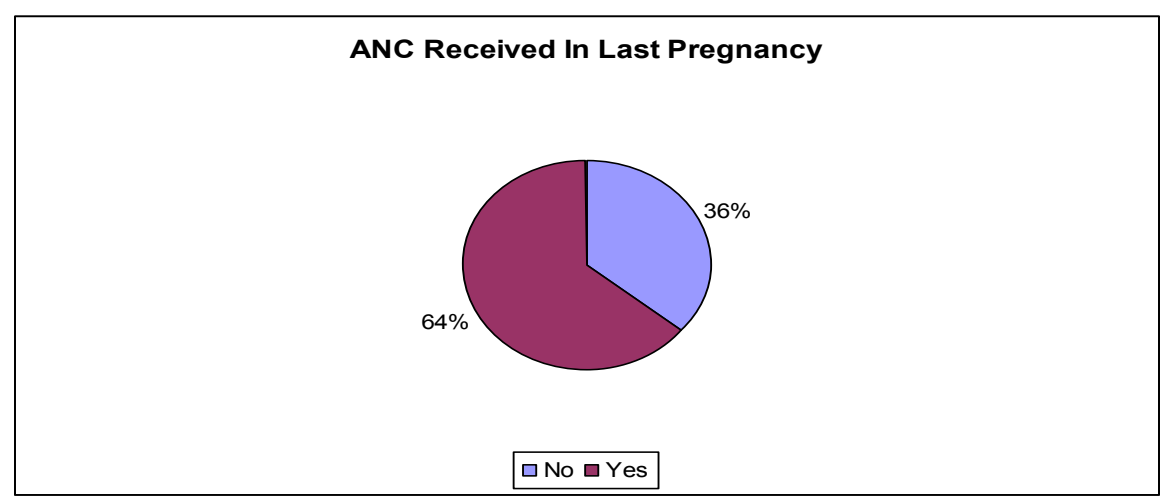

The pregnant women who reported visit of health facility were asked what were information were provided by service providers. The information obtained in this regard shows that $56 \%$ of the women said that they were told about signs of pregnancy complications followed by weighted when pregnant $(57.2 \%)$, height measured when pregnant $(35.8 \%)$, blood pressure taken when pregnant $(56.3 \%)$ and urine test when pregnant $(36.6 \%)$.

Access to mass media an important determinant of health care services during the pregnancy. Information collected in this regard. One in four respondents stated that they usually read newspaper/ magazine; similar percentage of the respondents $(25.1 \%)$ listen radio and a vast majority $(86 \%)$ of the respondents. Information on the access to mass media of the respondents was collected and is presented in Table 1. As information suggests over $81 \%$ of the respondents mentioned that they have access to mass media.

Table 1: Access to Mass Media

\begin{tabular}{cccc}
\hline \multirow{2}{*}{ Variables } & \multicolumn{3}{c}{ Total } \\
\cline { 2 - 4 } & & Frequency & Percentage (\%) \\
\hline Mass media access & Yes & 2881 & 81.2 \\
& No & 668 & 18.8 \\
Total & & 3549 & 100.0 \\
\hline
\end{tabular}

\subsection{Association between ANC and socio-economic and demographic characteristics}

Association between age and ANC were investigated through Chi-square analysis. The value of Chi-square (2.55) is significant at 5\% level of significance indicating that age of the respondents was an important determinant whether or not to receive ANC.

Table 2: Association between ANC and age

\begin{tabular}{cccc}
\hline \multirow{2}{*}{ Age in Years } & \multicolumn{3}{c}{ Antenatal Care Received } \\
\cline { 2 - 4 } & No & Yes & Total \\
\cline { 2 - 4 } & Frequency & Frequency & Frequency \\
\hline$<25$ years & 213 & 422 & 635 \\
$>=25$ years & 266 & 439 & 705 \\
\hline
\end{tabular}


Association between income and ANC was also investigated through Chi-square analysis. The value of chisquare $(7.1 ; \mathrm{p}>. \mathrm{o} 1)$ shows that there was a strong association between the income of the respondents and ANC received by the respondents.

Table 3: Association between Income and ANC

\begin{tabular}{cccc}
\hline \multirow{2}{*}{$\begin{array}{c}\text { Monthly Income } \\
\text { (TAKA) }\end{array}$} & \multicolumn{3}{c}{ Antenatal Care Received } \\
\cline { 2 - 4 } & No & Yes & Total \\
\cline { 2 - 4 } & Frequency & Frequency & Frequency \\
\hline$<5000$ & 255 & 283 & 538 \\
$5000-10000$ & 188 & 392 & 580 \\
$=>10000$ & 36 & 186 & 222 \\
\hline
\end{tabular}

The chi-square between education and ANC is significantly related suggesting that the higher the level of education the higher was the likelihood of receiving ANC during pregnancy because educated women were aware about the importance of ANC during pregnancy.

Table 4: Association between Education Level and ANC

\begin{tabular}{cccc}
\hline & \multicolumn{3}{c}{ Antenatal Care Received } \\
\hline Education level & No & Yes & Total \\
\cline { 2 - 4 } & Frequency & Frequency & Frequency \\
\hline No education & 272 & 307 & 579 \\
Primary & 159 & 286 & 445 \\
Secondary & 46 & 239 & 285 \\
Higher & 2 & 29 & 31 \\
Secondary+ & & & \\
\hline
\end{tabular}

Table 5 shows that the in recent years access to mass media like TV play significant role with ANC. Access to TV helps the respondents to aware about the importance of TV in disseminating information on ANC during the pregnancy. Besides information maternal and child health care its importance in the reduction of maternal and child mortality are highlighted in mass media which significantly helps the TV watchers to know what to during the pregnancy period. The Chi-square analysis shows that there was a strong association between TV watchers and ANC.

Table 5: Association between Mass Media and ANC

\begin{tabular}{cccc}
\hline Mass Media & \multicolumn{3}{c}{ Antenatal Care Received } \\
\cline { 2 - 4 } Access & No & Yes & Total \\
\cline { 2 - 4 } & Frequency & Frequency & Frequency \\
Yes & 390 & 794 & 1184 \\
no & 89 & 67 & 156 \\
\hline
\end{tabular}

\section{CONCLUSION}

This study indicates that overall knowledge about antenatal care was found to be better among women who had utilized antenatal care as compared to women who did not receive antenatal care. Women of reproductive age (15-49) need to recognize the importance of antenatal care and to receive such care in the community. Underlying this need, there is also a need to uplift the socio-economic status and literacy level through community based education. In particular there is need to increase reproductive health education, highlighting the importance of seeking antenatal care, and recognition of sign and symptoms and danger signs in pregnancy. Women are very much reluctant to select place of delivery and skilled personnel at delivery until they faced serious complications. Health seeking behavior is highly distinctive among the poor and the rich women. Findings of this study may have some policy implications, merit additional comment and recommendations that would help to Government to achieve improvement in maternal health.1. As in increase in both male and female education and literacy and consequently a probable raise in the social status can be expected result in improved maternal health in Bangladesh. Education may provide awareness for the use of ANC service. 2. Absence of major socio-cultural barriers must be capitalized by targeting all husbands and mother in laws to infuse the idea of delivery planning and make further improvement in the existing knowledge of women on early ANC, timing of first and all subsequent ANC visit. 3. Desired numbers of ANC by pregnant mothers from medically trained provider can be ensured. The availability of quality service providers and ANC provider in the service centers and can be ensured. 


\section{REFERENCES}

[1] A.Wagstaff and M.Claeson, The Millennium Development Goals for Health Rising to the Challenges, Washington DC: World Bank 2004.

[2] N. Chakraborty, M.A.Islam, R.I. Chowdhury, W.Bari and H.H.Akhter, Determinants of the use of maternal health services in rural Bangladesh, Health Promotion International, 18(4), 2003,327-337.

[3] World Health Organization (WHO), Maternal Mortality in 2000: Estimates Developed by WHO, UNICEF and UNFPA Geneva WHO 2004

[4] Ministry of Health and Family Planning Welfare (MOHFW), Bangladesh National Strategy for Maternal Health, Dhaka, Bangladesh: MOHFW, 2001.

[5] Z. Matthews, S, Mahendra, A. Kilaru, S Ganapathy, Antenatal care, care seeking and morbidity in rural Karnataka, India: results of a prospective study, Asia-Pacific Population Journal 16(2), 2001,11-28.

[6] WHO/UNICEF, Antenatal care in developing countries: promises, achievements and missed opportunities: an analysis of trends, levels and differentials, 1999-2001, World Health Organization, 2003, Geneva.

[7] A.M., Vanneste , C. Ronsmans, J. Chakraborty, A.A. Francisco ,Prenatal screening in rural Bangladesh: from prediction to care, Health Policy and Planning, 15(1),2000, 1-10.

[8] S.L. Gortmaker, The effects of Prenatal Care upon the Health of the Newborn, American Journal of Public Health 69,1979, 653-660. 